\title{
Extracellular vesicles are involved in oxidative stress and mitochondrial homeostasis in pulmonary arterial hypertension
}

\author{
Durairaj Sekar $^{1}$
}

Received: 9 April 2021 / Revised: 19 April 2021 / Accepted: 21 April 2021 / Published online: 20 May 2021

(C) The Japanese Society of Hypertension 2021

Pulmonary arterial hypertension (PAH) is an aggressive vascular condition characterized by elevated pulmonary artery pressure ( $>25 \mathrm{~mm} \mathrm{Hg}$ ), shortness of breath, and other symptoms [1]. Endothelial cell dysfunction, vascular cell hypertrophy, fibroblasts, and the immune system are associated with PAH $[2,3]$. Mitochondrial dysfunction and oxidative stress may induce PAH [4]. Although much progress has been made concerning the management of $\mathrm{PAH}$ in recent years, the exact molecular mechanism underlying the progression of PAH and potential therapeutic targets in cells remain unclear. Novel therapeutic targets have been identified, but their roles in treating PAH are still under investigation. On the other hand, extracellular vesicles (EVs) are secreted by cells and are made up of lipids that can be found in the extracellular space [5]. EVs contain nucleic acids and proteins that are associated with cellular and molecular functions in cells. Depending upon their biogenesis, size, and content, EVs can be categorized into different types, namely, exosomes, microvesicles, and apoptotic bodies, in cells. In general, functional characterization of EVs can reveal their clinical significance for disease progression and therapeutic targets. Characterizing EVs also helps to study cell communication signals in the pathological environment. EVs are secreted in response to the activation of various cellular growth factors, oxidative stress, inflammation, senescence, hypoxia, shear stress, cell death, bacterial endotoxin or exotoxins, etc. Many diseases are associated with EV-associated signaling pathways, which play an important role in determining therapeutic targets or biomarkers for PAH [6]. Recently, it has been revealed that exosomes play a very important role in the treatment of PAH in animal models [7]. From the above points, it is clear that EVs are important biomolecules that play a vital role in determining prognostic, diagnostic, and therapeutic targets.

Interestingly, a recent article published by Giana Blume Corssac et al. [8] in Hypertension Research showed that $\mathrm{PAH}$ induces the release of circulating EVs with oxidative content and alters redox and mitochondrial homeostasis in the brains of rats. The authors explained the role of EVs in $\mathrm{PAH}$ with respect to oxidative stress and mitochondrial homeostasis. They revealed a signaling pathway in which EVs are involved using a PAH animal model to assess oxidative stress and mitochondrial parameters in the brain as well as in the right ventricle (RV). They also observed upregulated expression of manganese superoxide dismutase, downregulated expression of hypoxia-inducible factor (HIF$1 \alpha)$ and the preservation of antioxidant enzyme expression in the RV and brain. Overall, the article explains the potential role of EVs in PAH and antioxidant defense mechanisms in protecting cells from disease progression. Figure 1 represents the protective effects of different signaling molecules during the development of PAH with EVs. The number of articles on EVs as biomarkers and therapeutic targets for PAH is very limited, and the authors of this paper briefly outlined the pathways that are significant to their studies, which will be beneficial for researchers who aim to understand the consequences and the role of EVs and oxidative stress in PAH. Currently, there is no effective treatment option or valid biomarker for the management of PAH, and it is necessary to identify therapeutic targets to treat this deadly disease. In addition, the number of studies in this research area is low; more clinical studies with larger samples will be useful in improving the treatment of PAH.
Durairaj Sekar

duraimku@gmail.com
Dental Research Cell and Biomedical Research Unit, Saveetha Dental College and Hospital, Saveetha Institute of Medical and Technical Sciences, Saveetha University, Chennai, India 
Fig. 1 The protective effects of different signaling molecules that occur during the development of pulmonary arterial hypertension (PAH) with extracellular vesicles (EVs)

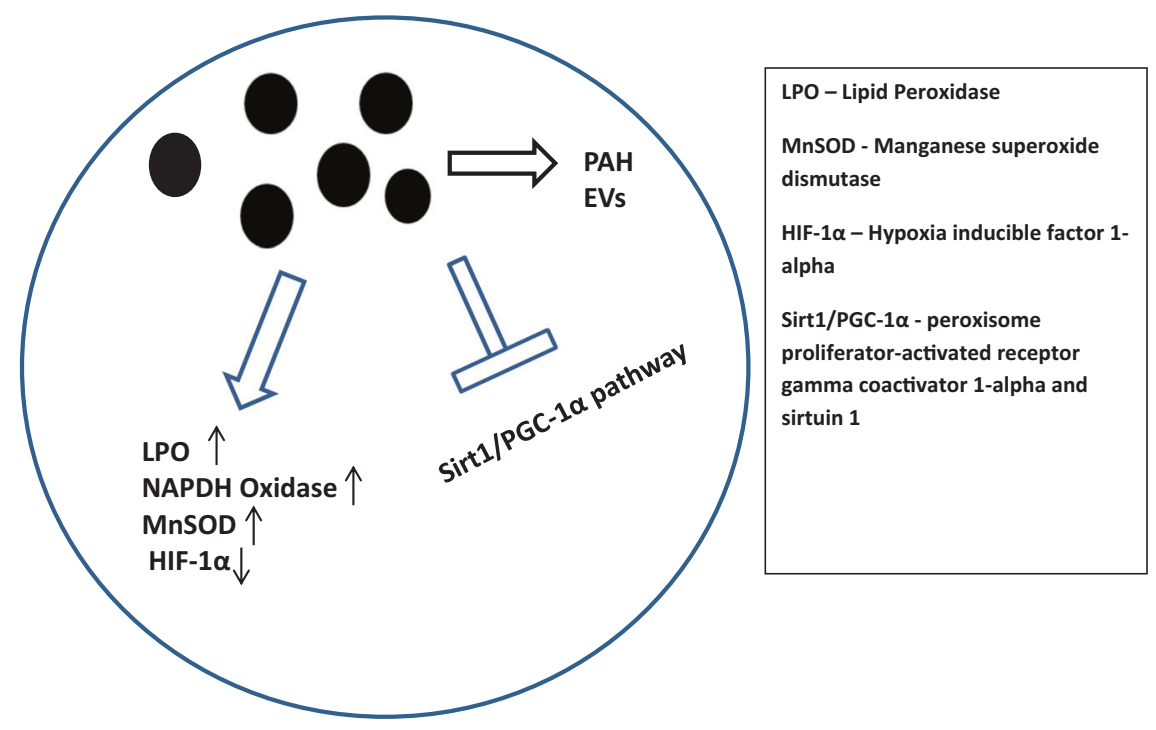

Acknowledgements DS is a recipient of the Extramural Grant (20190106/CMB/ADHOC/BMS) from the Indian Council of Medical Research (ICMR), Government of India, and their support is duly acknowledged.

\section{Compliance with ethical standards}

Conflict of interest The author declares no competing interests.

Publisher's note Springer Nature remains neutral with regard to jurisdictional claims in published maps and institutional affiliations.

\section{References}

1. Prins KW, Thenappan T. World Health Organization Group I Pulmonary Hypertension: epidemiology and pathophysiology. Cardiol Clin. 2016;34:363-74.

2. Tuder RM, Archer SL, Dorfmuller P, Erzurum SC, Guignabert C, Michelakis E, et al. Relevant issues in the pathology and pathobiology of pulmonary hypertension. J Am Coll Cardiol. 2013;62 (Suppl. 25):D4-D12.
3. Stratton MS, Farina FM, Elia L. Epigenetics and vascular diseases. J Mol Cell Cardiol. 2019;133:148-63.

4. Bello-Klein A, Mancardi D, Araujo AS, Schenkel PC, Turck P, de Lima Seolin BG. Role of redox homeostasis and inflammation in the pathogenesis of pulmonary arterial hypertension. Curr Med Chem. 2018;25:1340-51.

5. Doyle LM, Wang MZ. Overview of extracellular vesicles, their origin, composition, purpose, and methods for exosome isolation and analysis. Cells. 2019;8:727.

6. Simeone P, Bologna G, Lanuti P, Pierdomenico L, Guagnano MT, Pieragostino D, et al. Extracellular vesicles as signaling mediators and disease biomarkers across biological barriers. Int J Mol Sci. 2020;21:2514.

7. Ge L, Jiang W, Zhang S, Wang J, Xin Q, Sun C, et al. Mesenchymal stromal cell-derived exosomes attenuate experimental pulmonary arterial hypertension. Curr Pharm Biotechnol. 2020. https:// doi.org/10.2174/1389201022666201231113127.

8. Corssac GB, Bonetto JP, Cristina C-C, Cechinel LR, Zimmer A, Parmeggiani $\mathrm{B}$, et al. Pulmonary arterial hypertension induces the release of circulating extracellular vesicles with oxidative content and alters redox and mitochondrial homeostasis in the brain of rats. 2021. Hypertens Res. 2021. https://doi.org/10.1038/s41440-02100660-y. 